\title{
Inconsistency of Time-Symmetry Model
}

\author{
Amrit S. Šorli \\ Scientific research centre Bistra, Slovenija \\ sorli.bijective.physics@gmail.com \\ https://orcid.org/0000-0001-6711-4844 \\ Štefan Čelan \\ Scientific research centre Bistra, Slovenija \\ stefan.celan@bistra.si \\ https://orcid.org/0000-0003-3646-1469
}

\begin{abstract}
Can physical objects be in time-symmetry? Physical objects can only exist in a medium that has physical attributes, which means this medium is a type of energy. Is time energy? This article will show that time is not energy and there is no possibility that physical objects could be in time-symmetry. Physical objects only can be in symmetry in the time-invariant space in which they exist. In this perspective time measured with clocks is the result of the observer's measurement in the time-invariant space. The time-symmetry model is flawed.
\end{abstract}

Keywords: time, space, symmetry.

\section{Introduction}

The idea of 20th-century physics was that elementary particles can move forward and backward in space-time, which was considered the fundamental arena of the universe. Feynman has hypothesized that electron when moving backward in time is becoming positron. His idea of the symmetry between electron and positron in space-time was an example of the so-called "time-symmetry". Feynman has published his article on the theory of the positrons back in 1949 (1). From 1949 until now we do not have in physics any experimental confirmation that time is the 4 th dimension of space. The idea of time-symmetry needs rigorous re-examination that we will carry out in this article.

\section{Universal space is time-invariant}

Experimental physics is confirming that with clocks we measure the duration of material changes, i.e., motion in space. Based on experimental physics facts we have to admit that universal space is time-invariant in the sense that the duration of material change that in experimental physics is time, does not have any impact on space. We measure duration of material change or not, the physical properties of space will not be changed which means 
universal space is time-invariant (2). Time invariant universal space has a so-called "timeless configuration" $(3,4)$. In this space with timeless configuration, gravity is immediate and is the result of the quantum structure of time-invariant universal space (2).

\section{The model of time reversal symmetry is flawed}

The model of time reversal symmetry (T-symmetry) has no physical correspondence in the physical world. The equation of time symmetry has no physical meaning:

$$
T: t \rightarrow-t \quad(1) .
$$

Experimental research is confirming that time reversal symmetry is a model that has no bijective correspondence with the physical world (5). The discovery of positron particle does not prove that time-reversal symmetry exists. The scientific fact is that the electron and positron are both discovered and they both move and exist in the same universal space.

The model of space-time where past is represented as the negative time $-t$ and future is represented as the positive time $+t$ needs to be aligned with human observation and experimental results. In physics, we do not have a single proof that negative time exists and it is time we abandon this idea. The same is valid for the symmetry in time. There is no symmetry in time because there is no negative time $-t$. Elapsed time $t$ is not positive and is not negative, it has an absolute value:

$$
t=|t| \quad(2)
$$

\section{The model of time-invariant space-symmetry has physical existence}

The only symmetry that exists between physical objects is the symmetry in timeinvariant space. For example, in an EPR-type experiment, the electrons will have always the opposite spin. This is the measurable effect of the "space symmetry". Also, the energy of space, we call it today "superfluid quantum space" is in perfect symmetry with the mass of a given physical object accordingly to the equation:

$$
E=m c^{2}=\left(\rho_{E P}-\rho_{E m i n}\right) V
$$

where $E$ is the energy of the object, $m$ is the mass of the object, $\rho_{E P}$ is the Planck energy density of the space in the intergalactic space, $\rho_{E \min }$ is the energy density of the space in the centre of the object, and $V$ is the volume of the object (2). This formula is valid from the proton 
scale to the black hole scale. It describes the mass-energy equivalence principle extension on the universal space.

\section{Model of time-invariant space solves the "four-vector" puzzle}

In special relativity, the four-vector is introduced in order to unify space-time coordinates $\mathrm{x}, \mathrm{y}, \mathrm{z}$, and $\mathrm{t}$ into a single entity. The length of this four-vector, called the spacetime interval, is shown to be invariant, which means the same for all observers: $A=$ $\left(A^{0}, A^{1}, A^{2}, A^{3}\right)$, where $A^{0}$ as a temporal coordinate is $A^{0}=c t$. The so-called "temporal coordinate" is a product of time $\mathrm{t}$ as the duration of motion and light speed $\mathrm{c}$. The four-vector can be positive or negative and depends on the direction of motion in future or in past:

$$
d \tau= \pm \sqrt{d x^{\mu} d x_{\mu}}
$$

where $\tau$ is proper time [6].

The idea of motion into past or into future is questionable because it leads to the logical inconsistency where the sum of positive four-vector and negative four-vector is zero:

$$
\sqrt{d x^{\mu} d x_{\mu}}+\left(-\sqrt{d x^{\mu} d x_{\mu}}\right)=0 \quad \text { (5) [6]. }
$$

This means that the value of the space-time interval in the Minkowski manifold from A to B and back from $\mathrm{B}$ to $\mathrm{A}$ is zero which seems wrong. In our model of time-invariant space a given physical object can move only in a $\mathbb{C}^{4}$ space and not in time that is the duration of motion [2]. The value of the four-vector $A=\left(A^{1}, A^{2}, A^{3}, A^{4}\right)$ in a $\mathbb{C}^{4}$ space is always absolute; you cannot move into past or into future. You can only move in time-invariant space. There is no negative time $-t$ and there is no negative four-vector. The puzzle is solved.

Rovelli has proposed the existence of physical time is an allusion [7]. Our research has confirmed his idea and added an important fact: time is an illusion until it is not measured. When measured by the observer, time enters existence as the duration. In the physical universe, the time has only a mathematical existence; time is the numerical order of changes, i.e., motion in space. Once this "fundamental time" is measured by the observer the "emergent time" enters existence. This emergent time is the duration we measure with clocks [8]. 


\section{Conclusions}

Experimental physics is confirming that time is the duration of a material change, i.e., motion in space. The duration that we measure with blocks cannot be positive or negative, elapsed time is always an absolute value $|t|$. The time-symmetry model has no single experimental support and should be abandoned in the name of physics progress.

\section{References:}

1. R. P. Feynman, The Theory of Positrons, Phys. Rev. 76, 749 (1949) https://doi.org/10.1103/PhysRev.76.749

2. Šorli, A.S. \& Čelan Š. Time-invariant Superfluid Quantum Space as the Unified Field Theory, Reports in Advances of Physical Sciences, Vol. 4, No. 3 (2020) 2050007, https://doi.org/10.1142/S2424942420500073 (2021)

3. Gomes, H. Timeless Configuration Space and the Emergence of Classical Behavior. Found Phys 48, 668-715 (2018). https://doi.org/10.1007/s10701-018-01721

4. Gomes, H. Quantum gravity in timeless configuration space, Class. Quantum Grav. 34235004 (2017) 10.1007/s10701-018-0172-1

5. Müller, Clemens, Guan, Shengwei, Vogt, Nicolas, Cole, Jared H, \& Stace, Thomas M, Passive on-chip superconducting circulator using a ring of tunnel junctions, Physical review letters, 120(21), 213602 (2018) https://doi.org/10.1103/PhysRevLett.120.213602

6. Jose M. Ripalda, Time reversal and negative energies in general relativity (2010) arXiv:gr-qc/9906012

7. Andrew Jaffe, The illusion of time, Nature, Nature 556, 304-305 (2018) https://doi.org/10.1038/d41586-018-04558-7

8. Fiscaletti, D., Sorli, A. Perspectives of the Numerical Order of Material Changes in Timeless Approaches in Physics. Found Phys 45, 105-133 (2015). https://doi.org/10.1007/s10701-014-9840-y 